\title{
Highly Anisotropic Dirac Cones in Epitaxial Graphene Modulated by an Island Superlattice
}

\author{
S. Rusponi, ${ }^{1}$ M. Papagno, ${ }^{2}$ P. Moras, ${ }^{2}$ S. Vlaic, ${ }^{1}$ M. Etzkorn, ${ }^{1}$ P. M. Sheverdyaeva, ${ }^{2,3}$ D. Pacilé, ${ }^{4}$ \\ H. Brune, ${ }^{1}$ and C. Carbone ${ }^{2}$ \\ ${ }^{1}$ Institute of Condensed Matter Physics (ICMP), Ecole Polytechnique Fédérale de Lausanne (EPFL), CH-1015 Lausanne, Switzerland \\ ${ }^{2}$ Istituto di Struttura della Materia-Consiglio Nazionale delle Ricerche, Basovizza, Trieste, Italy \\ ${ }^{3}$ Sinctrotrone Trieste SCpA, SS14 Km 163.5, Trieste, Italy \\ ${ }^{4}$ Dipartimento di Fisica, Universitá della Calabria, and INFN-Gruppo collegato di Cosenza, 87036 Arcavacata di Rende (CS), Italy
} (Received 19 July 2010; revised manuscript received 13 October 2010; published 7 December 2010)

\begin{abstract}
We present a new method to engineer the charge carrier mobility and its directional asymmetry in epitaxial graphene by using metal cluster superlattices self-assembled onto the moiré pattern formed by graphene on $\operatorname{Ir}(111)$. Angle-resolved photoemission spectroscopy reveals threefold symmetry in the band structure associated with strong renormalization of the electron group velocity close to the Dirac point giving rise to highly anisotropic Dirac cones. We further find that the cluster superlattice also affects the spectral-weight distribution of the carbon bands as well as the electronic gaps between graphene states.
\end{abstract}

Graphitic materials have attracted strong scientific interest because they exhibit exotic phenomena such as superconductivity or the anomalous quantum Hall effect [1]. Graphene (gr) is the building block of these materials; it is wrapped up into fullerenes, rolled up into carbon nanotubes, or stacked into 3D graphite. It presents a model system to investigate the influence of many-body interactions on the electron dynamics in these materials. In addition, the exceptional electronic mobility makes graphene a candidate material for next generation electronic devices [2]. Freestanding graphene is a zero-gap semiconductor. Because most electronic applications require a gap between valence and conduction bands, considerable effort has been spent to induce and control the opening of such a band gap [3-6].

A related, and for applications equally relevant, issue is the ability to tailor the band dispersion. The speed with which information can be transmitted along a graphene layer depends on the charge carrier group velocity. Close to the $K$ points, the $\pi$ bands of freestanding graphene have a linear dispersion well described by the relativistic Dirac equation for massless neutrinos. The resulting Dirac cones are trigonally warped due to the chiral nature of graphene charge carriers in the equivalent $A$ and $B$ carbon sublattices [7]. The ability to increase and tailor this anisotropy would open a manifold of new applications. Several theoretical studies suggest that this goal can be reached by applying an external periodic potential with nanometer period giving rise to highly anisotropic Dirac cones [8-11].

Epitaxial graphene layers grown on lattice mismatched close-packed metal substrates, such as $\operatorname{Pt}(111)$ [12], $\operatorname{Ir}(111)$ [13], or $\mathrm{Ru}(0001)$ [14], represent ideal model systems to test such predictions. The lattice mismatch between graphene and substrate gives rise to moiré structures creating a potential with the required nanometer period. These potentials themselves have been seen to generate small band gaps since they break the carbon sublattice symmetry by the different adsorption sites of the two $C$ species, very much as in graphene on the $(6 \sqrt{3} \times 6 \sqrt{3})$-reconstructed buffer layer on $4 \mathrm{H}-\mathrm{SiC}(0001)$ $[4,5]$. However, no significant group velocity asymmetry has been reported up to date.

Here we demonstrate with angle-resolved photoemission spectroscopy (ARPES) that an Ir cluster superlattice grown on the $\mathrm{gr} / \operatorname{Ir}(111)-(9.32 \pm 0.15 \times 9.32 \pm 0.15)$ moiré structure $[13,15]$ gives rise to significant group velocity and Dirac cone asymmetries. We attribute this to a much stronger periodic potential caused by the cluster superlattice than by the moiré itself. $\mathrm{H}$ decorated $\mathrm{gr} / \operatorname{Ir}(111)$ has been reported to give rise to band gap opening but not to the Dirac cone asymmetries reported here [6]. We thereby confirm theoretical predictions and present a new way to tailor the directionality of carrier mobility. In addition, the cluster superlattice changes the band structure symmetry from sixfold to threefold, increases the gap size at the mini-Brillouin zone boundaries, and is responsible for the widening of the band gap at the Dirac points.

$\operatorname{The} \operatorname{Ir}(111)$ crystal has been prepared by repeated cycles of $\mathrm{Ar}^{+}$ion sputtering at room temperature and at $1000 \mathrm{~K}$, followed by annealing to $1500 \mathrm{~K}$. The graphene layer has been grown by exposing the $\operatorname{Ir}(111)$ surface at $1300 \mathrm{~K}$ to ethylene $[15,16]$. The decomposition of ethylene is selflimiting; therefore, only a single graphene layer forms, which we confirmed by ARPES. The Ir cluster superlattice has been grown by evaporating $0.15 \mathrm{ML}$ Ir [one monolayer (ML) is defined as one atom per Ir substrate atom] at a substrate temperature of $375 \pm 25 \mathrm{~K}$. Under these conditions, exactly one Ir cluster with a mean cluster size of 13 atoms is formed per hep region of the gr/Ir(111)- $(9.32 \times 9.32)$ moiré $[13,16]$. Ir has been evaporated from a current heated thin film plate with a cross section of $0.5 \mathrm{~mm} \times 0.1 \mathrm{~mm}$. The deposition flux has 
been adjusted to $2.0 \times 10^{-4} \mathrm{ML} / \mathrm{s}$ and calibrated by the core level intensity measured for Ir deposition on a $\mathrm{Ag}(111)$ crystal. This calibration has been cross-checked with STM in a different UHV chamber. ARPES data have been collected at the vacuum ultraviolet photoemission beam line of the Elettra synchrotron radiation facility in Trieste with a photon energy of $120 \mathrm{eV}$. This energy is close to the Cooper minimum of the photoionization cross section of the Ir $5 d$ states and thereby minimizes the Ir background signal. ARPES spectra have been taken with the sample temperature being fixed at $100 \mathrm{~K}$ and with a SCIENTA R-4000 hemispherical electron analyzer which allows spectra to be recorded simultaneously within an angular aperture of $30^{\circ}$.

Figure 1 compares ARPES intensity maps at energies close to the Fermi level $\left(E_{F}\right)$ for $\operatorname{gr} / \operatorname{Ir}(111)$, without and with the superlattice of Ir islands on top. The bright feature at the $K$ point shows the cross section of the graphene $\pi$ band forming the Dirac cone. One can clearly see its replicas situated at the moiré reciprocal vectors $\mathbf{G}_{m}$ [17]. Matrix elements in the photoemission process limit the detection of Dirac cone replicas to the $\mathbf{k}$-space region close to the $K$ point of graphene and affect the azimuthal dependence of the photoemission intensity [18,19]. Close inspection reveals that all visible replicas have similar intensity and shape for $\operatorname{gr} / \operatorname{Ir}(111)$, while the cluster superlattice introduces a threefold symmetry (in both cases, some of the replicas are masked by the strong Ir $d$-band intensity at the edge of the Brillouin zone). The unperturbed moiré superpotential has close to sixfold symmetry. This is in agreement with the structural similarity between fcc and hcp compared with atop stacking areas, which is also reflected in the small apparent height difference in STM constant current images between the first two compared with the last stacking area [16]. In contrast, the Ir clusters are seen to accentuate the difference between the hcp and fcc regions. At the chosen deposition temperature the clusters are situated exclusively on the hcp regions where they rehybridize carbon orbitals from $s p_{2}$ to $s p_{3}$
$[13,20]$. This changes the superpotential symmetry from hexagonal to trigonal and perturbs the photoemission spectral-weight distribution suppressing three out of the six replicas.

More important than the change in photoemission intensity $[21,22]$ is the modification of the graphene band structure by the potential exerted by the cluster superlattice. The most prominent effect is the renormalization of the group velocity expressed in highly anisotropic Dirac cones. This is seen in Fig. 2 comparing the ARPES intensity acquired for $\mathrm{gr} / \operatorname{Ir}(111)$ and $\mathrm{Ir} / \mathrm{gr} / \operatorname{Ir}(111)$ along $\Gamma K$ and along the perpendicular direction passing through the $K$ point $p \Gamma K$ (see schematic representations in Fig. 1). Fits of the momentum distribution curves (MDCs) with double Lorentzian functions are shown in Fig. 2(c). We focus on the energy region close to the apex of the Dirac cone, where the effect of the superlattice potential is strongest. Since the linear dispersion of the $\pi$ band is modified in proximity of the Bragg planes, we restrict our analysis to energies $E-E_{F}>$ $-0.5 \mathrm{eV}$. For $\operatorname{Ir} / \mathrm{gr} / \operatorname{Ir}(111)$ we find $v_{\Gamma K}=4.90 \pm$ $0.06 \mathrm{eV} \AA$ and $v_{p \Gamma K}=2.90 \pm 0.05 \mathrm{eV} \AA$, corresponding to an anisotropy of $\Delta v / v_{p \Gamma K}=70 \pm 5 \%$. This value is 12 times larger than the anisotropy expected for unperturbed graphene due to the trigonal warping. The tightbinding approximation up to third-nearest neighbors (TB3 model) for freestanding graphene gives $v_{\Gamma K}=5.5 \mathrm{eV} \AA$, $v_{p \Gamma K}=5.2 \mathrm{eV} \AA$, and $\Delta v / v_{p \Gamma K}=5 \%$ [23]. The anisotropy for $\operatorname{gr} / \operatorname{Ir}(111)$ is with $\Delta v / v_{p \Gamma K}=16 \pm 2 \%\left(v_{\Gamma K}=\right.$ $\left.5.00 \pm 0.05 \mathrm{eV} \AA, v_{p \Gamma K}=4.29 \pm 0.06 \mathrm{eV} \AA\right)$ larger than this, showing the effect of the moiré; however, it is significantly smaller than the one of the cluster superlattice.

The group velocity renormalization is expected to be more effective for charge carriers moving perpendicularly to the largest corrugation of the superpotential $[8,10,11]$. The large anisotropy in $\operatorname{Ir} / \mathrm{gr} / \operatorname{Ir}(111)$ is created by the much stronger reduction of $v_{p \Gamma K}$, by a factor of 2 when compared with theory [23] and by even more when compared with

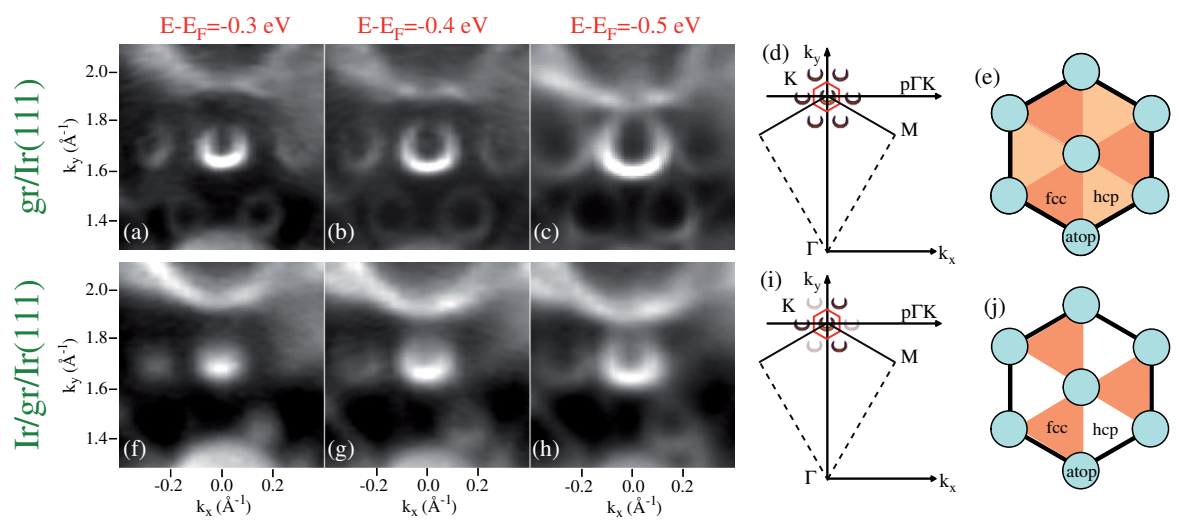

FIG. 1 (color online). ARPES intensity constant energy maps. Upper line: gr/Ir(111); lower line: $\operatorname{Ir} / g r / \operatorname{Ir}(111)$. (a)-(c) and (f)-(h) Mini-Brillouin zone around the $K$ point. (d),(i) Schematic representations of the principal Dirac cone with its replicas. The red hexagon represents the mini-Brillouin zone generated by the moiré superpotential. (e),(j) Moiré scheme of the stacking areas of gr rings centered on hcp, fcc, and atop substrate sites. The colors display the enhancement of the potential difference between the faulted and unfaulted half of the moiré unit cell by the Ir cluster superlattice. 

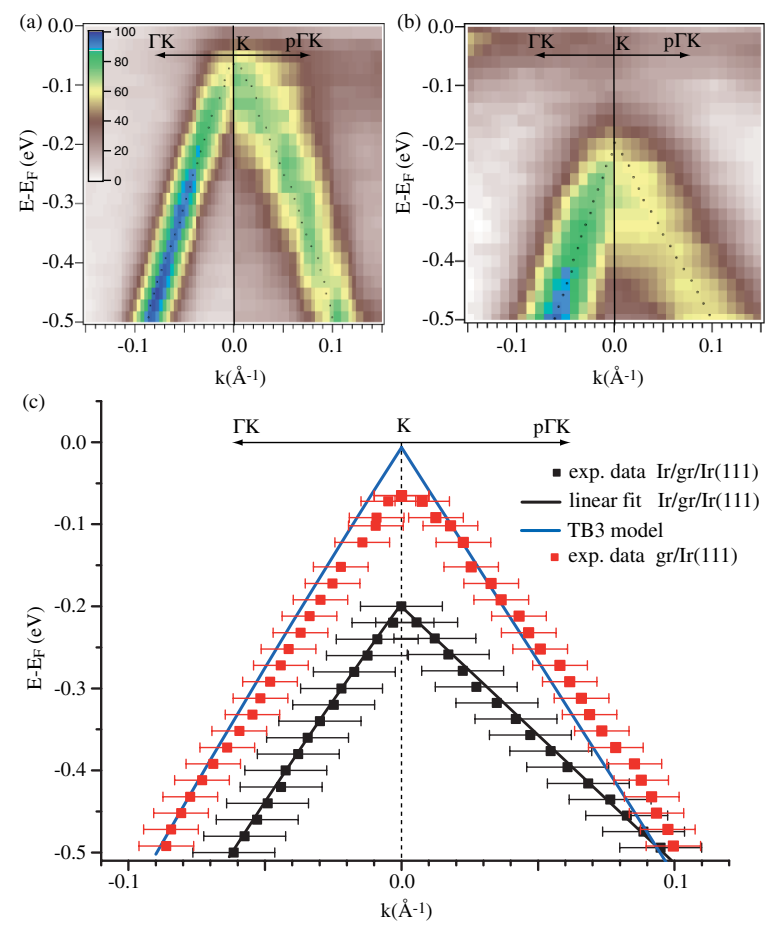

FIG. 2 (color online). (a) ARPES intensity of the $\pi$ band of graphene in $\mathrm{gr} / \operatorname{Ir}(111)$, around the $K$ point, along the $p \Gamma K$ and the $\Gamma K$ directions. A linear dispersion is observed along both directions. (b) ARPES intensity of the $\pi$ band of graphene in $\mathrm{Ir} / \mathrm{gr} / \operatorname{Ir}(111)$ along the same two directions. In (a) and (b) the dots represent the result of the MDCs fitting. (c) The fitted band positions. Red and black squares were determined by fitting the MDCs extracted from (a) and (b). The $k$-vector error bars are determined as the HWHM of the Lorentzian fits of the MDCs and are representative of the spread of the band intensity. Error bars in energy $(20 \mathrm{meV})$ are not displayed. The blue curves represent the band dispersion predicted by the TB3 model, whereas black lines are the linear fits of the experimental band for the $\operatorname{Ir} / \mathrm{gr} / \operatorname{Ir}(111)$. In $\operatorname{Ir} / \mathrm{gr} / \operatorname{Ir}(111)$ the profile is highly asymmetric compared to TB3 and gr/Ir(111).

magnetotransport measurements finding $v=7.3 \mathrm{eV} \AA$ [24,25], while $v_{\Gamma K}$ reduces by less than $25 \%$.

Additional evidence of band structure modification by the cluster superpotential is seen by the minigaps opening at the border of the mini-Brillouin zone. Figures 3(a) and 3(b) display ARPES maps along the $p \Gamma K$ direction for $\mathrm{gr} / \operatorname{Ir}(111)$ without and with cluster superlattice. The corresponding energy distribution curves are shown in Figs. 3(c) and 3(d). The minigap width $\left(\Delta_{p \Gamma K}\right)$ for $k_{x}=$ $\pm 0.14 \AA^{-1}$ increases from $240 \pm 20 \mathrm{meV}$ for $\mathrm{gr} / \operatorname{Ir}(111)$ [17] to $330 \pm 20 \mathrm{meV}$ for $\operatorname{Ir} / \mathrm{gr} / \mathrm{Ir}(111)$. The enlarging of the minigap is in agreement with theoretical calculations [8]. However, at odd with theory, Fig. 3 excludes the existence of a new Dirac point at the $M$ point of the mini-Brillouin zone [9].

In addition, the cluster superlattice enlarges the graphene band gap $\left(E_{g}\right)$ at the $K$ point. For $\operatorname{gr} / \operatorname{Ir}(111)$ the graphene $\pi$ photoemission intensity along $p \Gamma K$ disperses linearly toward $E_{F}$; see Fig. 2. Its summit is with
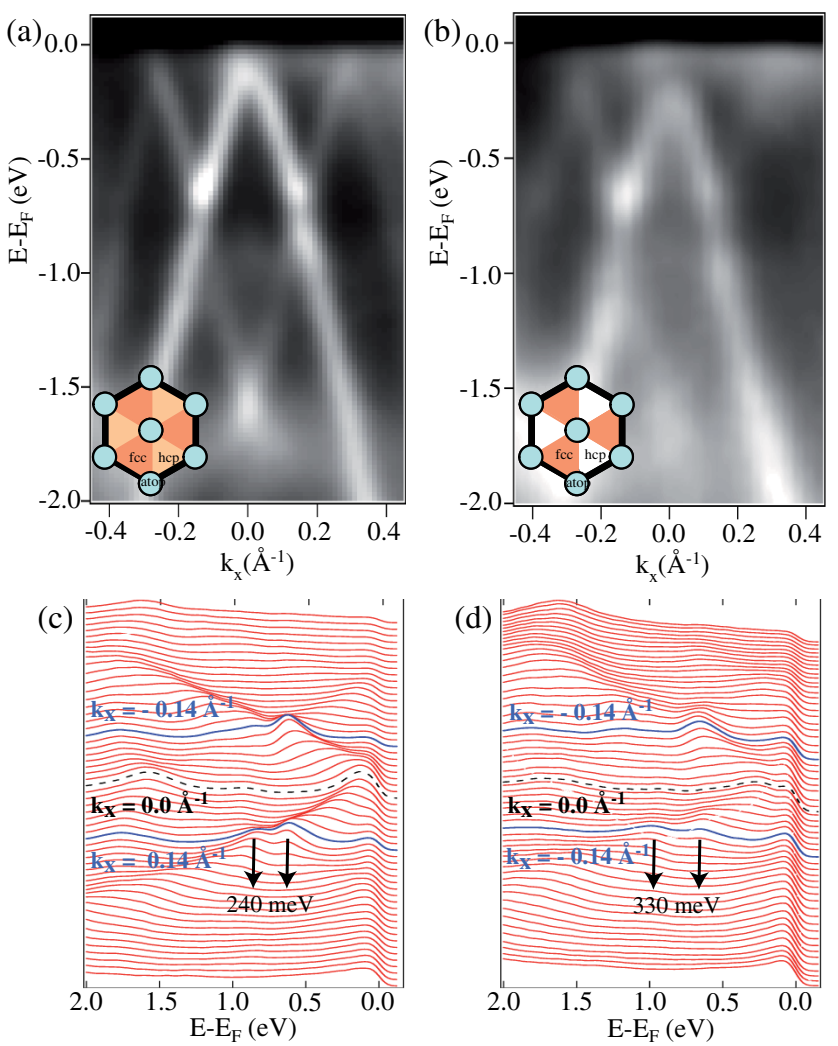

(d)
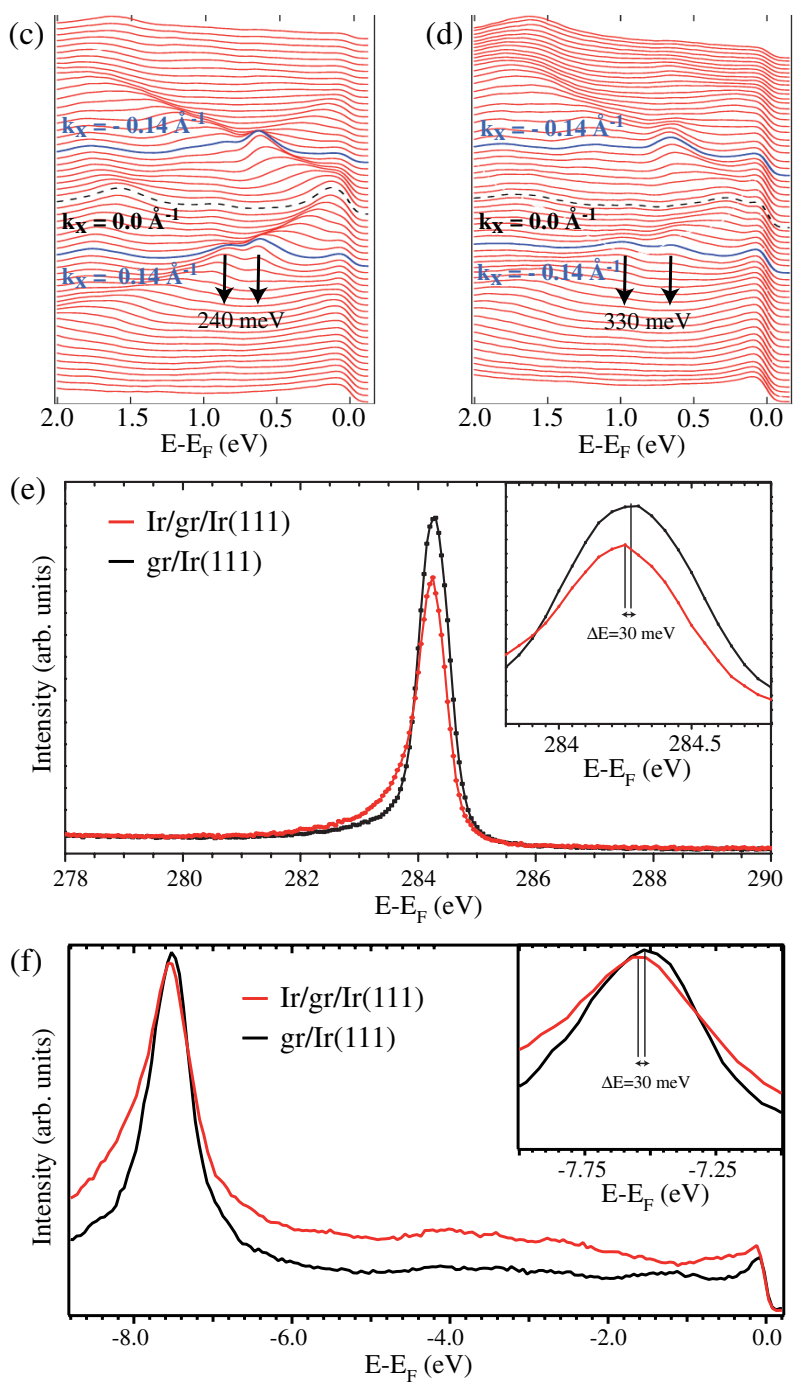

FIG. 3 (color online). (a),(b) ARPES intensity of the graphene $\pi$ band along the $p \Gamma K$ direction in $\operatorname{gr} / \operatorname{Ir}(111)$ (a) and $\mathrm{Ir} / \mathrm{gr} / \mathrm{Ir}(111)$ (b). (c),(d) Corresponding energy distribution curve plots. (e) $C$ - $1 s$ core levels for an Ir coverage of $\theta=0.15 \pm$ 0.05 ML. Inset: Zoom on the $C-1 s$ peak showing a shift of $30 \pm$ $30 \mathrm{meV}$ after the cluster growth. (Normal emission, photon energy 450 eV.) (f) Energy distribution spectra from $\operatorname{gr} / \operatorname{Ir}(111)$ and $\operatorname{Ir} / \operatorname{gr} / \operatorname{Ir}(111)$ measured at the $M$ point of the second Brillouin zone. Inset: The top of the $\sigma$ band shifts by $30 \pm 30 \mathrm{meV}$ after the cluster growth. 
$E_{\pi}=-70 \pm 20 \mathrm{meV}$ very close to $E_{F}$, in good agreement with previous results [6]. The Ir cluster superlattice shifts the $\pi$ summit down to $E_{\pi}=-200 \pm 20 \mathrm{meV}$, while the $\pi^{*}$ band stays above $E_{F}$. Because the $\pi^{*}$ band is not observed in the ARPES spectra, we can only estimate the band gaps of both systems from their $E_{\pi}$ values. Without assumptions on the Dirac energy we can say that $E_{g} \geq$ $-E_{\pi}$ giving $E_{g-\operatorname{gr} / \operatorname{Ir}(111)} \geq 70 \mathrm{meV}$ for $\operatorname{gr} / \operatorname{Ir}(111)$ and $E_{g-\operatorname{Ir} / \mathrm{gr} / \mathrm{Ir}(111)} \geq 200 \mathrm{meV}$ for $\operatorname{Ir} / \mathrm{gr} / \operatorname{Ir}(111)$. More precise estimates can be derived by assuming a value for $E_{D}$ for $\mathrm{gr} / \operatorname{Ir}(111)$. For $\operatorname{Ir} / \mathrm{gr} / \operatorname{Ir}(111) E_{D}$ might be different due to a possible chemical shift that can be estimated from the core level and conduction band energy shifts. The $C-1 s$ level shifts down by $30 \pm 30 \mathrm{meV}$ [see Fig. 3(e)], and the top of the $\sigma$ band at the $M$ point of the second Brillouin by the same amount [see Fig. 3(f)]. Thus cluster deposition shifts the Dirac point down by $30 \mathrm{meV}$. Assuming charge neutrality for $\mathrm{gr} / \operatorname{Ir}(111)[6,20]$, one finds $E_{g-\operatorname{gr} / \operatorname{Ir}(111)}=$ $140 \mathrm{meV}$ and $E_{g-\mathrm{Ir} / \mathrm{gr} / \mathrm{Ir}(111)}=340 \mathrm{meV}$. For an interval of reasonable values of $-35 \mathrm{meV} \leq E_{D} \leq 50 \mathrm{meV}$ for $\mathrm{gr} / \operatorname{Ir}(111)$, we find that the cluster superlattice increases the band gap by factors from 3.9 to 1.8 .

The lifting of the conduction-valence band degeneracy at the $K$ point requires breaking the equality between the $A$ and $B$ sublattices of graphene [3,5,6]. In $\mathrm{gr} / \operatorname{Ir}(111)$ both sublattices are close to identical in the atop stacking areas of the moiré, where both atoms are located on the fcc and hcp substrate hollow sites. They are very close to degenerate also in the fcc and hcp moiré stacking areas, where $C_{A}$ atoms are on top of Ir atoms and $C_{B}$ atoms on hcp hollows, respectively, $C_{B}$ on top Ir and $C_{A}$ on fcc hollows, due to the very weak interaction of $\mathrm{C}$ atoms with the Ir substrate [20]. Consequently, only a small gap at the $K$ point is expected in $\mathrm{gr} / \operatorname{Ir}(111)\left(E_{g-\mathrm{gr} / \mathrm{Ir}(111)} \leq 140 \mathrm{meV}\right.$ in our measurements; see also the existence of a small gap before hydrogenation in Ref. [6]). This changes significantly by the cluster superlattice. The clusters nucleate only on the hcp stacking areas of the moiré where they strongly bind to the $C_{A}$ atoms located on fcc substrate hollows. As a consequence, also the $C_{B}$ atoms bind more strongly to the Ir substrate atoms below them, as suggested in Refs. [6,20], thus leading to the observed asymmetry between $A$ and $B$ sublattices [26].

In conclusion. we have experimentally demonstrated the possibility to induce anisotropy in the energy dispersion of the Dirac cones of a graphene layer by the periodic potential caused by a cluster superlattice. This observation confirms previous theoretical predictions. Moreover, the large anisotropies of group velocities together with the opening of the band gap between the $\pi-\pi^{*}$ bands represent a fundamental step toward band gap engineering needed for graphene-based electronic circuits.

This work has been supported by the European Science Foundation (ESF) under the EUROCORES Program EuroGRAPHENE, the EUROCORES SONS 2 SANMAG (FN 20S221-112613), and by the Swiss National Science
Foundation (Grants No. FN 200020-120493 and No. FN 200020-129934). The authors thank M. Grioni for helpful discussions.

[1] A. K. Geim and K.S. Novoselov, Nature Mater. 6, 183 (2007).

[2] Y.M. Lin, C. Dimitrakopoulos, K. A. Jenkins, D. B. Farmer, H. Y. Chiu, A. Grill, and P. Avouris, Science 327, 662 (2010).

[3] G. W. Semenoff, Phys. Rev. Lett. 53, 2449 (1984).

[4] S. Y. Zhou, G. H. Gweon, A. V. Fedorov, P. N. First, W. A. D. Heer, D. H. Lee, F. Guinea, A. H.C. Neto, and A. Lanzara, Nature Mater. 6, 770 (2007).

[5] S. Kim, J. Ihm, H. J. Choi, and Y. W. Son, Phys. Rev. Lett. 100, 176802 (2008).

[6] R. Balog et al., Nature Mater. 9, 315 (2010).

[7] P. R. Wallace, Phys. Rev. 71, 622 (1947).

[8] C. H. Park, L. Yang, Y.W. Son, M. L. Cohen, and S. G. Louie, Nature Phys. 4, 213 (2008).

[9] C. H. Park, L. Yang, Y.W. Son, M. L. Cohen, and S. G. Louie, Phys. Rev. Lett. 101, 126804 (2008).

[10] C. H. Park, Y. W. Son, L. Yang, M. L. Cohen, and S. G. Louie, Phys. Rev. Lett. 103, 046808 (2009).

[11] L. Brey and H. A. Fertig, Phys. Rev. Lett. 103, 046809 (2009).

[12] T. A. Land, T. Michely, R. J. Behm, J. C. Hemminger, and G. Comsa, Surf. Sci. 264, 261 (1992).

[13] A. T. N'Diaye, S. Bleikamp, P. J. Feibelman, and T. Michely, Phys. Rev. Lett. 97, 215501 (2006).

[14] S. Marchini, S. Günther, and J. Wintterlin, Phys. Rev. B 76, 075429 (2007).

[15] J. Coraux, A. T. N'Diaye, C. Busse, and T. Michely, Nano Lett. 8, 565 (2008).

[16] A. T. N'Diaye, J. Coraux, T. N. Plasa, C. Busse, and T. Michely, New J. Phys. 10, 043033 (2008).

[17] I. Pletikosic, M. Kralj, P. Pervan, R. Brako, J. Coraux, A. T. N'Diaye, C. Busse, and T. Michely, Phys. Rev. Lett. 102, 056808 (2009).

[18] E. L. Shirley, L. J. Terminello, A. Santoni, and F. J. Himpsel, Phys. Rev. B 51, 13614 (1995).

[19] M. Mucha-Kruczyński, O. Tsyplyatyev, A. Grishin, E. McCann, V.I. Fal'ko, A. Bostwick, and E. Rotenberg, Phys. Rev. B 77, 195403 (2008).

[20] P. J. Feibelman, Phys. Rev. B 77, 165419 (2008).

[21] J. Voit, L. Perfetti, F. Zwick, H. Berger, G. Margaritondo, G. Grüner, H. Höchst, and M. Grioni, Science 290, 501 (2000).

[22] F. Clerc, C. Battaglia, H. Cercellier, C. Monney, H. Berger, L. Despont, M. G. Garnier, and P. Aebi, J. Phys. Condens. Matter 19, 355002 (2007).

[23] S. Reich, J. Maultzsch, C. Thomsen, and P. Ordejón, Phys. Rev. B 66, 035412 (2002).

[24] K. S. Novoselov, A. K. Geim, S. V. Morozov, D. Jiang, M. I. Katsnelson, I. V. Grigorieva, S. V. Dubonos, and A. A. Firsov, Nature (London) 438, 197 (2005).

[25] Y. Zhang, Y. W. Tan, H. L. Stormer, and P. Kim, Nature (London) 438, 201 (2005).

[26] The $\mathrm{C}$ rehybridization may be responsible for the appearance of the small shoulder observed in the core level spectrum. 